\title{
Recent advances in understanding the fungal G-protein-coupled receptors
}

\author{
Kalaivani Nadarajah*, Ilakiya Sharanee Kumar, Vanee Sangapillai, Nurfarahana Syuhada Omar \\ School of Environmental and Natural Resource Sciences, Faculty of Science and Technology, Universiti Kebangsaan \\ Malaysia, 43600 Bangi, Selangor, Malaysia. \\ Email: vani@ukm.edu.my
}

\begin{abstract}
Transmission of extracellular signal across the plasma membrane into the cells of organisms is impossible without cell surface receptors. One of the most broadly studied receptor is the G-protein coupled receptor. This receptor is coupled with heterotrimeric $G$ proteins with $\alpha, \beta$ and $y$ subunits that perceives external stimuli and transduces the signal into the cell for suitable physiological and biochemical responses. They have also been reported as potential receptors to sense light and fatty acids, but their exact mechanism remains unclear in fungi. Signalling and regulation via $\mathrm{G}$ proteins has been extensively studied in various models including pathogenic fungi. Fungal GPCRs are broadly required in fungal defence stimulation, vegetative growth, and pathogenicity mechanism. This review aims to highlight the research in fungal GPCRs including classification, physiological roles, mechanisms of action and signalling in GPCR function. Through fungal genome sequencing, mammalian GPCRs have been identified apart from fungal-specific GPCRs which adds another dimension to the classification. The deorphanisation of unclassified fungal GPCRs is necessary to further understand their role in fungi. While the mechanism of action has been well documented in mammals, the glucose and pheromone sensing are the only two well mapped systems in yeast. However, we are yet to ascertain if there are any additional mechanisms of signalling at work in fungi. Further we endeavour to compare and contrast between the eukaryotic GPCRs in various aspects of functionality. Through the information derived we hope to determine the gaps in knowledge and by so doing determine the future directions of GPCR research in fungi.
\end{abstract}

Keywords: G-Protein coupled receptors; eukaryotic; pathogenic fungi; plant- fungal interaction

\section{INTRODUCTION}

G protein and G-protein coupled receptors (GPCRs) are one of the largest protein families in eukaryotes and are widely discovered on the exterior layers of all cells of eukaryotic organisms (Premont and Gainetdinov, 2007). As the name implies, GPCRs are coupled with heterotrimeric G-protein with 3 different subunits namely $\alpha, \beta$ and $\gamma$, which can bind to GTP and GDP. The GPCRs play an integral role at the molecular level of biochemical reaction of almost all multicellular organisms (Drake et al., 2006). The seven membrane-spanning domains are regarded as the unique structural architecture of GPCR which has a wide range of ligand binding specificity (Rosenbaum et al., 2009; Tuteja, 2009). The range in ligand binding specificity is a consequence of the first cytoplasmic loop formation in GPCR which changes the conformation post ligand binding, bringing about the third loop which is responsible for $G$ protein interactions and signal transmission (Stefan and Blumer, 1994).

Various ligands such as $\mathrm{Ca}^{2+}$, fatty acids, odorants, amino acids, proteins, peptide pheromone, nucleotides and steroids (Maller, 2003) can activate GPCR for further intracellular signalling. The GPCRs signalling system is vital for most physiological processes in eukaryotes including vision, taste, smell, nervous system cardiovascular, endocrine, and reproductive functions. The involvement of GPCR in these important processes made them a well-studied major target for drugs development (Overington et al., 2006).

Although GPCRs have been extensively studied in animal and plant systems (Urano and Jones, 2014), genome studies have identified many GPCRs in fungi where some have shown identity to mammalian like GPCRs. The fungal specific GPCRs include nutrient sensors (Gpr1), cAMP like receptors, pheromone receptors sensing peptide pheromones (Ste2), Stm1-like nitrogen sensors (Stm1), pheromone receptors sensing lipid modified peptide pheromones (Ste3), microbial opsins (Nop-1 and Orp-1), and glucose sensor (Gpr4) (Han et al., 2004a; Han et al., 2004b). The fungal specific GPCR such as pheromone sensing and glucose sensing receptors are well characterised in yeast. However, the fungal specific receptors do not follow exactly the standard GRAFS system and have been further classified into 6 classes, which will be discussed further.

Fungal GPCRs are required for various signalling and regulation of vegetative growth, mating, and development of the organism, pathogenicity and regulation of biosynthetic pathways. Through numerous sensing 
mechanisms such as pheromone, nutrient, nitrogen, carbon (glucose and amino acid sensing) and light sensing, GPCR initiates signalling cascades specific to the bound ligand. A GPCR known as GPCR3, found in the entomopathogenic fungi, Beauveria bassiana, was shown to be responsible for nutrient sensing, stress and developmental responses (Ying et al., 2013). Besides, a seven-transmembrane protein known as Stm1, in Schizosaccharomyces pombe was required for nitrogen starvation signals under nitrogen-deficient condition (Chung et al., 2001). Glucose sensing G protein-coupled receptors, Gpr1 in Saccharomyces cerevisiae is the first GPCR discovered that senses nutrient in fungi (Dijck, 2009). Two more receptors, Ste2 and Ste3 characterised in S. cerevisiae are important in pheromone signalling (Chen et al., 1997). In Neurospora crassa, the GPR-4 GPCR (NcGPR-4) senses carbon sources by coupling to the Group I Ga GNA-1, which operates upstream of the cAMP signalling pathway to regulate growth and asexual development.

In addition, GPCRs are also involved in fungal-plant interactions. To infect the host plant, the fungal pathogen recognises various signal molecules or ligands from plant cells through the signalling from fungal GPCR. Homologs of the fungal specific receptor, CAMP receptors from Dictyostelium, and a steroid receptor MPR were found in the pathogenic fungi, Magnaporthe grisea (Kulkarni et al., 2005). This is accompanied by the dicovery of PTH11, a novel class of fungal receptor that facilitates surface recognition during appressorium formation for pathogenesis in M. grisea (Kulkarni et al., 2005).

Studies on Aspergillus nidulans revealed that GPCRs and regulators of G protein signalling (RGSs) functions in controlling development of appressorium and the attachment of spore on the host (Yu, 2006). In N. crassa, ten (10) GPCRs sequences were predicted and the newly identified sequences were responsible for extracellular CAMP signalling pathway during chemotaxis and multicellular development through the encoded proteins that control cAMP levels (Galagan et al., 2005). Aspergillus flavus was identified to encode fifteen (15) putative GPCRs which controlled germination, biosynthesis of secondary metabolites, sporulation, and the production of spores at high and low cell densities (Affeldt et al., 2014).

As mentioned above, the function of GPCR is largely dependent on binding and interaction between components of this protein with other proteins. Therefore, this protein-protein interaction is largely dependent on the protein structure.

\section{Structure and classification of fungal GPCR}

Over the years, many fungal specific GPCRs have been identified in various fungal species and have been classified into six classes based on the similarities in sequence and function (Xue et al., 2008). The first class of fungal GPCR (Class I) consists of pheromone receptors for alpha-factor pheromone (Ste2-like). This class of GPCR senses alpha factor pheromones and interacts with $\mathrm{G}$ proteins to initiate the signalling cascades that leads to mating between haploid-a and alpha cells. The first ever discovery of the function of this receptor was shown in Ste2 receptor in S. cerevisiae (Burkholder et al., 1985). Different regions within the fungal pheromone receptor Ste2 function in different ways to perform the role of Ste2. The N-terminal domain promotes the oligomerisation of Ste2 (Uddin et al., 2012) whereas, the C-terminal domain is involved in the negative regulation of the receptor (Schandel et al., 1994).

Class II of fungal specific GPCR includes pheromone receptors for a-factor pheromone (Ste3-like). GPCR in this class senses a-factor pheromone and transmits signalling cascades to conduct functions similar to Class I fungal GPCR. One of the earliest studies on this class of receptor revealed that the STE3 gene in $S$. cerevisiae is likely to be a membrane protein and encodes a 7TM region like other GPCRs which concludes that it's a GPCR that is involved in pheromone signalling for afactor pheromones (Hagen et al., 1986). The fungal pheromone mating-factor receptors Ste 2 and Ste 3 (Class I and Class II) were originally classified into Class D of the general GPCR classification system. The other classes are discussed later.

The next class of fungal GPCRs (Class III) are carbon sensors or specifically known as homologs of glucose sensor Gpr1. The GPCR carbon receptor senses glucose, sucrose and methionine and activates cAMP signalling through a-subunit Gpa2 (Miwa et al., 2004; Maidan et al., 2005). Glucose sensing in S. cerevisiae is the most well represented mechanism in carbon sensing. Carbon sources are required for spore germination in $A$. nidulans and Botrytis cinerea and possibly many other fungi (d'Enfert, 1997; Doehlemann et al., 2006).

Class IV of fungal GPCR consists of nutrient sensing GPCRs. Nutrient sensing GPCRs initiate signalling cascades for downstream signalling response to regulate virulence, metabolism and morphogenesis (Bölker, 1998; Lengeler et al., 2000; Versele et al., 2001). This class of receptor can be further subdivided into 3 classes. The first class contains GPCRs that resemble yeast Gpr1 receptor, the second class contains GPCRs closely related to the GprD receptor of $A$. nidulans and the third class exhibits putative nutrient sensing GPCRs that shows homology to Sch. pombe Stm1 receptor (Dijck, 2009) which is required for nitrogen starvation signals under nitrogen-deficient condition (Chung et al, 2001).

The cAMP receptor-like (CRL) constitutes class $\mathrm{V}$ of fungal GPCRs and was first characterised in ascomycete fungi, $N$. crassa which was known as Gpr1 (not to be confused with yeast Gpr1). It was thought to regulate the fruiting body formation and mating in fungi (Krystofova and Borkovich, 2006). The CRLs share similar homology to four cAMP receptors (cAR1-cAR4) and three cAMP receptor-like proteins (CrlA-CrIC) in Dictyostelium discoideum, and Arabidopsis thaliana GCR1 (Klein et al., 1988; Plakidou-Dymock et al., 1998; Raisley et al., 2004). However, this class of receptor is absent in the yeasts $S$. cerevisiae and Sch. pombe. 
Class VI of GPCRs are microbial opsin which are represented by NOP-1 opsin from $N$. crassa (Bieszke et al., 1999a) and ORP-1 opsin-related protein from Leptosphaeria maculans (Idnurm and Howlett, 2001). Opsin genes are divided into 2 different types, whereby type I are microbial opsins and type II are animal opsins (Spudich et al., 2000). GPCRs from Class VI are not to be confused with animal opsins as these two groups of opsins do not share similar sequence homology. However, the members of this class share similarity to archaeal opsins. NOP-1 has been shown to bind all-trans retinal in vitro and may have signalling functions in vivo (Bieszke et al., 1999b). Apart from these six classes, there are also a few putative GPCRs that remained unclassified (Li et al., 2007). Table 1 shows the list of well characterised fungal specific GPCRs found in filamentous fungi and yeast according to their respective classes.

Apart from the fungal specific GPCRs, mammalianlike GPCRs were also found in fungi. The classification of metazoan GPCR is based on the ubiquitous GRAFS system (Fredriksson et al., 2003; Fredriksson and Schioth, 2005; Lagerström et al., 2008) which stands for Glutamate, Rhodopsin, Adhesion, Frizzled and Secretin (de Mendoza et al., 2014). The mammalian GRAFS families are exceptionally characterised in Metazoa, but they were not represented in fungi until the most recent study on fungal genome which revealed that four mammalian families of GPCRs, except Secretin, were in fact present in fungi (Krishnan et al., 2012).

Rhodopsin-like receptors are from Class A GPCRs and regarded as the largest and the most widespread group of GPCR. It has been sub-divided into 19 subfamilies (A1- A19) (Sgourakis et al., 2005). These groups include hormones, neurotransmitters and light receptors which transduces external stimuli by interacting with $G$ proteins. Rhodopsin-like receptors are characterised with short $\mathrm{N}$-terminal domains and interact with a broad variety of ligands (Krishnan et al., 2012). Three fungal species namely Allomyces macrogynus (Blastocladiomycota), Batrachochytrium dendrobatidis and Spizellomyces punctatus (Chytridiomycota) were found to contain 12 novel sequences of Rhodopsin family (Krishnan et al., 2012).

The second largest GPCRs are from the Adhesion family (Class B) with 33 members in humans (Fredriksson and Schioth, 2005). This receptor has long N-terminal with different functional domains and is classified into eight main groups (I-VIII) (Lagerström et al., 2008). In fungi, 30 novel Adhesion family members were found to be distributed among 22 species of the phylum Ascomycota and one in $A$. macrogynus from the phylum Blastocladiomycota (Krishnan et al., 2012). The GPCRs from Secretin family also constitute Family B of GPCR and are found in human and animals but yet to be dicovered in fungi (Miller et al., 2012)

The next class of GPCR is from Glutamate family (Class C) and is well-known as the metabotropic glutamate receptors (mGluRs) which includes GABA-B receptors, taste receptors, olfactory receptors and a few putative pheromone receptors coupled to the G-protein
Go (VRs and Go-VN) (Chaudhari et al., 2000; Heinbockel et al., 2007; Tuteja, 2009; Padgett et al., 2010). The Nterminal of this receptor is long and functions as endogenous ligand binding site (Krishnan et al., 2012). Based on a study conducted by Krishnan et al. (2012), 96 putative Glutamate receptors were found in 4 fungal species from three different phyla. A total of 78 GPCRs were found in $A$. macrogynus which belongs to phylum Blastocladiomycota. Meanwhile 14 GPCRs were identified in Sepsophis punctatus, 3 GPCRs in $B$. dendrobatidis phylum Chytridiomycota and 1 GPCR was found in Rhizopus oryzae (Zygomycota). However, none were found in the phyla Ascomycota and Basidiomycota up till now.

Frizzled-receptors (Class F) are receptors for Wnt protein, an important secreted glycoprotein (Rao and Kühl, 2010) in tissue polarity and cell signalling (Lagerström et al., 2008). This receptor has CRD_FZ domain with 10 conserved cysteine residues (Krishnan et al., 2012). Frizzled proteins and the genes that encode them have been identified across diverse species of animals from sponges to humans (Huang et al., 2004). Interestingly, two new Frizzled receptor sequences were found in S. punctatus (Krishnan et al., 2012). One interesting thing to note here is that although none of the mammalian like GRAFS families were found in Ascomycota and Basidiomycota, they are the only fungal phylum to have fungal specific GPCRs. The identification of mammalian-like GPCRs in fungi is indeed a great breakthrough in fungal GPCR research; however, functional studies should be conducted to have a better understanding on these receptors in fungal biological functions.

Unclassified G-proteins are known as orphan GPCRs. The ligands of these orphan GPCRs are unidentified and their physiological role are yet to be determined (Tang et al., 2012). A total of 16 GPCRs were identified in $A$. nidulans, but their ligands are unknown (Xue et al., 2008). In M. grisea, 76 GPCR-like proteins have been identified based on the genome sequence analysis (Kulkarni et al., 2005). More comprehensive studies on these orphan GPCRs in fungi may lead to the discovery of more signalling mechanisms that regulate different physiological responses. Besides, this existing classification system needs to be reconstructed to incorporate the fungal specific GPCR and mammalian like GPCR into a single integrated fungal GPCR classification system.

\section{Physiological roles of GPCR in fungi}

The interaction between cells with their environment is essential for the organism to conduct various functions for its survival. Cell surface receptors function as sensors to facilitate interaction between the cell and the environment. G protein-coupled receptors (GPCRs) are responsible in facilitating intracellular physiological processes by transducing extracellular signals into the cell. In fungi, G-proteins aid in regulating the cellular 
Malays. J. Microbiol. Vol 14(6) Special Issue 2018, pp. 611-623

DOI: http://dx.doi.org/10.21161/mjm.1461823

Table 1: List of well characterised fungal specific GPCRs in filamentous fungi and yeast according to respective classes.

\begin{tabular}{|c|c|c|c|c|c|c|c|}
\hline \multirow[t]{2}{*}{ Type of fungi } & \multirow[t]{2}{*}{ Species } & \multicolumn{2}{|c|}{ Pheromone receptor } & \multirow{2}{*}{$\begin{array}{l}\text { Carbon sensor } \\
\text { (Class III) }\end{array}$} & \multirow{2}{*}{$\begin{array}{l}\text { Nutrient sensor } \\
\text { (Class IV) }\end{array}$} & \multirow{2}{*}{$\begin{array}{l}\text { cAMP receptor-like } \\
(\text { Class V) }\end{array}$} & \multirow{2}{*}{$\begin{array}{l}\text { Microbial opsin } \\
\text { (Class VI) }\end{array}$} \\
\hline & & Ste2 like (Class I) & $\begin{array}{l}\text { Ste3 like } \\
\text { (Class II) }\end{array}$ & & & & \\
\hline \multirow{6}{*}{ Filamentous fungi } & N. crassa & PRE-2 & PRE-1 & Gpr 4 & Gpr5, Gpr6 & GPR1, GPR2, GPR3 & NOP-1, ORP-1 \\
\hline & A. nidulans & GprB & GprA & $\begin{array}{l}\text { GprC, GprD } \\
\text { GprE }\end{array}$ & $\begin{array}{l}\text { GprF, } \\
\text { AN5720 }\end{array}$ & $\begin{array}{l}\text { GprH, Gprl } \\
\text { AN8262 }\end{array}$ & AN3361 \\
\hline & C. neoformans & - & Ste3a/Ste3a, Cpr2 & & Gpr2, Gpr3 & Gpr4, Gpr5 & Ops1 \\
\hline & M. grisea & MGG_04711 & MGG_06452 & MGG_08803 & $\begin{array}{l}\text { MGG_04698MG } \\
\text { G_02855 }\end{array}$ & MGG_06738 & MGG_09015 \\
\hline & U. maydis & - & Pra1, Pra2 & - & $\begin{array}{l}\text { UM06006 } \\
\text { UM01546 }\end{array}$ & UM03423 & $\begin{array}{l}\text { UM02629 } \\
\text { UM04125 }\end{array}$ \\
\hline & C. cinerea & - & $\begin{array}{l}\text { Rcb1, Rcb2 } \\
\text { Rcb3 }\end{array}$ & - & $\begin{array}{l}\text { CC1G_07132 } \\
\text { CC1G_04180 }\end{array}$ & $\begin{array}{l}\text { CC1G_02288 } \\
\text { CC1G_02310 }\end{array}$ & - \\
\hline \multirow{3}{*}{ Yeast } & S. cerevisiae & Ste2 & Ste3 & Gpr4 & $\begin{array}{l}\text { SCRG01312 } \\
\text { SCRG02823 } \\
\text { SCRG00179 }\end{array}$ & - & - \\
\hline & S. pombe & Mam2 & Mam3 & Git3 & Stm1 & - & - \\
\hline & C. albican & Ste2 & Ste3 & Gpr1 & $\begin{array}{l}\text { CAWG02899 } \\
\text { CAWG06059 } \\
\text { CAWG02686 }\end{array}$ & - & - \\
\hline
\end{tabular}


Malays. J. Microbiol. Vol 14(6) Special Issue 2018, pp. 611-623

DOI: http://dx.doi.org/10.21161/mjm.1461823

Table 2: The differences of GPCR in fungi and other eukaryotes.

\begin{tabular}{|c|c|c|c|}
\hline Kingdom & Fungi & Animal & Plant \\
\hline $\begin{array}{l}\text { The requirement of GPCR in } \\
\text { signalling }\end{array}$ & GPCR-dependent & & GPCR-independent \\
\hline G-protein subunits & $\begin{array}{l}\text { Scarce } \\
2 \mathrm{Ga} \text { (yeast)/3Ga (filamentous fungi), } \\
1 \mathrm{G} \beta \\
1 \mathrm{~Gy} \text { (filamentous fungi)/ >1 Gy (in some } \\
\text { fungal species) }\end{array}$ & $\begin{array}{l}\text { Abundant } \\
23 \mathrm{G \alpha} \\
5 \mathrm{G} \beta \\
12 \mathrm{~Gy}\end{array}$ & $\begin{array}{l}\text { Scarce } \\
\text { Single } G \alpha \text { and } G \beta \text { subunits and } 2 \text { canonical } G y \\
\text { subunits }\end{array}$ \\
\hline Functions & $\begin{array}{l}\text { Growth, asexual \& sexual development, and } \\
\text { virulence }\end{array}$ & $\begin{array}{l}\text { Regulates diverse cellular } \\
\text { responses to the majority of } \\
\text { neurotransmitters and hormones }\end{array}$ & $\begin{array}{l}\text { Stomatal aperture control, fungal defense, oxidative } \\
\text { stress, development, sugar perception and } \\
\text { phytochrome/cryptochrome-mediated responses }\end{array}$ \\
\hline Effectors & $\begin{array}{l}\text { cAMP-dependent and mitogen-activated } \\
\text { protein kinase and adenylyl cyclases }\end{array}$ & $\begin{array}{l}\text { Adenylyl cyclases and other well- } \\
\text { known effectors }\end{array}$ & $\begin{array}{l}\text { Protein interactors may function similar to effectors in } \\
\text { animal and fungi. }\end{array}$ \\
\hline Ligand & Different ligands stimulate different GPCR & $\begin{array}{l}\text { Chosen ligand can stimulate GPCR } \\
\text { for different intracellular signalling }\end{array}$ & Ligands inhibit the inhibitor \\
\hline
\end{tabular}


functions of vegetative growth, conidiation, infection, structure differentiation and pathogenicity (Liu et al., 2007) and to carry out these functions, specific sensing mechanisms are being carried out by fungal GPCRs.

Fungal GPCRs are especially important in pheromone sensing in fungi to attract their mates. In ascomycetes and basidiomycetes, the signalling mechanism for the pheromone sensing starts with the detection of pheromones from the opposite mate by the cell surface 7TM GPCRs (pheromone receptors). Following pheromone binding, these receptors are activated to trigger the downstream-signalling pathways for the mating process (Xue et al., 2008). In addition, GPCRs have been reported to sense nutrients in some fungal species. The Gpr1 G protein-coupled receptor in $S$. cerevisiae functions as an upstream component in the cAMP-PKA pathway (Yun et al., 1997; Xue et al., 1998). Lorenz et al (2000) revealed that Gpr1 G protein-coupled receptor regulates pseudohyphal differentiation in $S$. cerevisiae. Additionally, the activation of cAMP synthesis by glucose and sucrose was shown to be mediated by this receptor (Yun et al., 1998). GPCRs also serve as a sensor for amino acid in fungi. For example, in Candida albicans, Gpr1 act as methionine sensor to control filamentation (Maidan et al., 2005).

A nutrient sensing GPCR, Stm 1 protein in Sch pombe is needed for a proper recognition of nitrogen starvation signals. Under nutritionally deficient conditions, the Stm 1 protein forces Sch. pombe cells to cease cell proliferation by meiosis (Chung et al., 2007). Opsin, a member of the GPCR rhodopsin Family A, serves as a sensory receptor in light sensing which helps in the developmental stage specifically in mating and sporulation (Idnurm and Heitman, 2005). Based on a recent study on $L$. maculans, opsin protein can build an electrochemical transmembrane gradient of proton by forming of proton pump which suggests that opsins in fungi may conduct a similar mechanism as opsins in Archaea (Waschuk et al., 2005).

GPCR might also be a potential receptor for free fatty acids (FFAs) in fungi although fatty acid sensors are not reported to date in fungi. FFAs have been demonstrated to be involved in the developmental process of fungus. For instance, oxylipins in $A$. nidulans, functions as a signalling molecule to promote the communication between fungus and host (Tsitsigiannis et al., 2006). Novel GPCR candidates have been characterised in both A. nidulans and Cryptococcus neoformans and it is proposed that these novel GPCR candidates may sense fatty acids (Xue et al., 2008). However, the mechanism by which these GPCRs can sense the FFAs remains unknown.

Ga subunits have conserved the signal transduction pathways governing mating, filamentous growth, and virulence. One $\mathrm{Ga}$ subunit, Gpa3 in the basidiomycete Ustilago maydis is important for both virulence and mating. In Cryphonectria parasitica, the causative agent of chestnut blight, virulence is regulated via a G-protein linked signal transduction pathway. A hypovirus that infects this fungal pathogen has $C P G-1$ gene that requires $\mathrm{Ga}$ subunits for mating, asexual sporulation and melanin production (Alspaugh et al., 1997). The appressoria formation of $M$. oryzae is initiated by $G$ protein $\alpha$ subunit MAGB which is triggered by MAP kinase following the activation of adenylate cyclase protein MAC 1 (Choi and Dean et al., 1997).

\section{Mechanism of action involving GPCR signalling}

\section{Glucose sensing mechanism}

Glucose is a simple sugar that serves as important energy source in all living organisms. In fungi, glucose is utilised in various metabolic processes such as germination, (Beyer et al., 2004), growth (Daynes et al., 2008) and fermentation (Panagiotou et al., 2008). To transport glucose inside the cell, fungi have developed various mechanisms to sense the glucose. Glucose sensing via GPCR signalling has been vastly studied in yeast (Bahn et al., 2007). Generally, the GPCR signalling starts with binding of ligand which activates GPCR to undergo conformational change to activate the dissociation of G-protein for the transmission of the intracellular signal. In $S$. cerevisiae, the glucose molecules act as ligands that bind to glucose sensing GPCR receptor (Gpr1) which in turn activates it (Xue et al., 1998) to undergo conformational change. This promotes the exchange of GDP to GTP at Gpa2 $\alpha$ subunit and its dissociation from the unknown $\beta y$ subunits. The Gpa2 is thought to stimulate the adenylyl cyclase (Cdc35/Cyr1) in S. cerevisiae which activates the cAMPdependent protein kinase (PKA) pathway for various developmental and mating processes (Figure 1a) (Versele et al., 2001). In other eukaryotes however, the common mechanism for glucose sensing through GPCR signalling is yet to be discovered. The above pathway involving GPCR Gpr1 in fungi sets an example for discovery of similar proteins and pathways in other eukaryotes.

\section{Pheromone sensing mechanism}

In living organisms, mating is an essential physiological process to ensure the survival of the species. Many organisms use substances known as pheromones to attract their mates (Gomez-Diaz and Benton, 2013). Fungi are not an exception in this matter. Again, the pheromone sensing mechanism in fungi is studied based on the model species $S$. cerevisiae. The yeast cells produce two types of mating factors known as a-factor and $\alpha$-factor (peptide pheromone) which act as ligands that bind to pheromone receptor Ste2 and Ste3 (Jones and Bennett, 2011). Following the binding of pheromone, pheromone receptor changes their conformation from an inactive $R$ state to an active $R^{\star}$ state which stimulates the GDP-GTP exchange at Gpa1 a subunit. Even though the sequences of Ste2 and Ste3 are dissimilar, both activate the same Gpa1 for GDP-GTP exchange when they are in $\mathrm{R}^{*}$ state (Xue et al., 2008). The Gpa1 will then be dissociated from Ste4 and Ste18 dimer ( $\beta$ y subunits). In 
most of the signalling pathways, both the dissociated $\mathrm{Ga}$ and $\mathrm{G} \beta \mathrm{y}$ communicate with effectors for downstream signalling. However, in $S$. cerevisiae pheromonesignalling pathway, Gßy complex plays a central role in inducing the downstream pheromone signalling cascades, where Ste 4 and Ste18 aid in the transmission of the signal to Ste20 that activates MAP-kinase cascade consisting of Ste11, Ste7 and Fus3 (Versele et al., 2001), leading to successful mating (Figure $1 \mathrm{~b}$ ). Hence, cells that do not have the GBy complex are unable to conduct mating responses (Whiteway et al., 1989). In addition to this model, a study on yeast proposed that $\mathrm{G} \alpha$ dissociates from Gßy dimer and moves into endosome to activate an endosome-localised phosphatidylinositol 3-kinase (PI3K) composed of four subunits including a catalytic subunit Vps34 and a regulatory subunit Vps15. Ga-GTP then binds to Vsp34 to activate PI3K activity producing phosphatidylinositol 3-phosphate, which serves as a binding site to recruit proteins to the endosome. Ga-GDP can also bind to $V p s 15$, which resembles a $G \beta$ subunit, without activating $\mathrm{PI} 3 \mathrm{~K}$. It is proposed that $\mathrm{Ga}$ may undergo cycles of GTP binding and hydrolysis at the endosome, which causes $\mathrm{Ga}$ to alternate binding between the two subunits of PI3K (Koelle, 2006). a) Glucose sensing GPCR

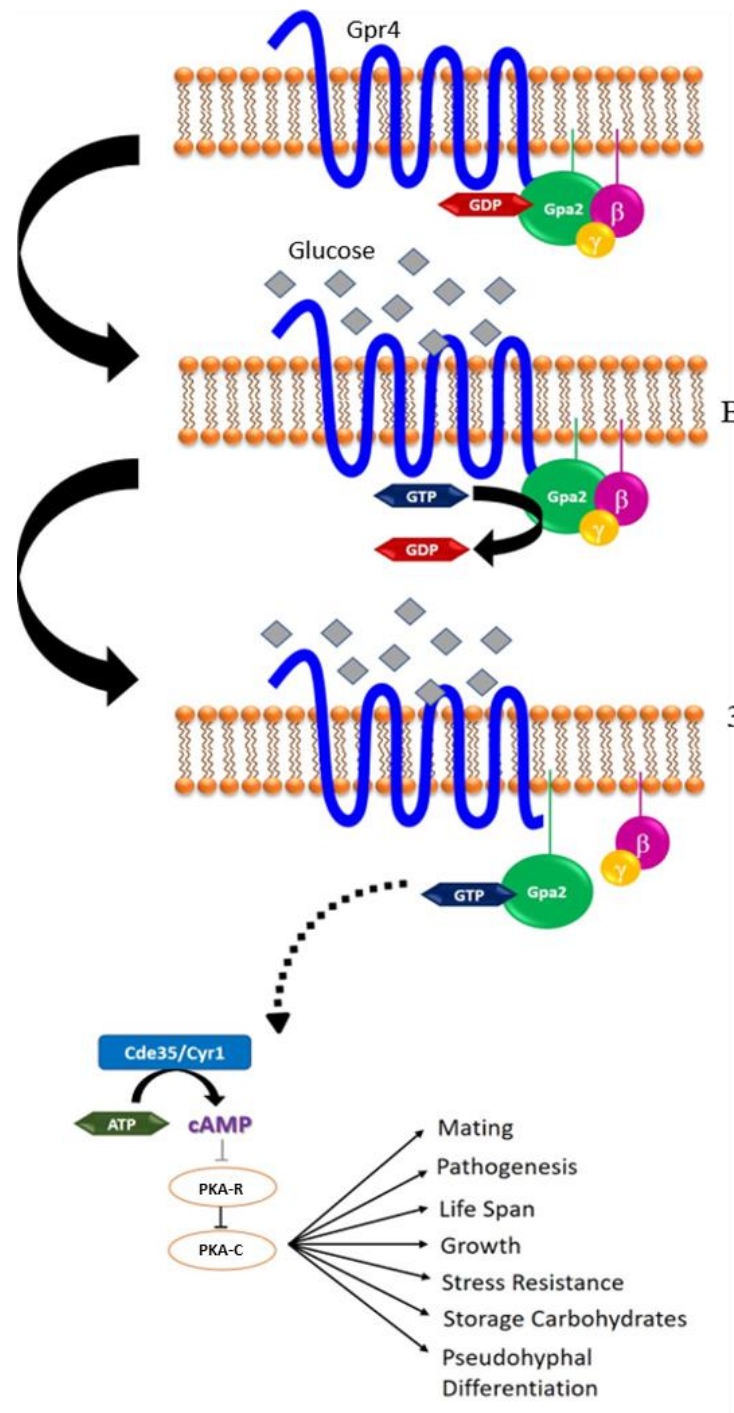

b) Pheromone-sensing GPCR

1. Inactive State
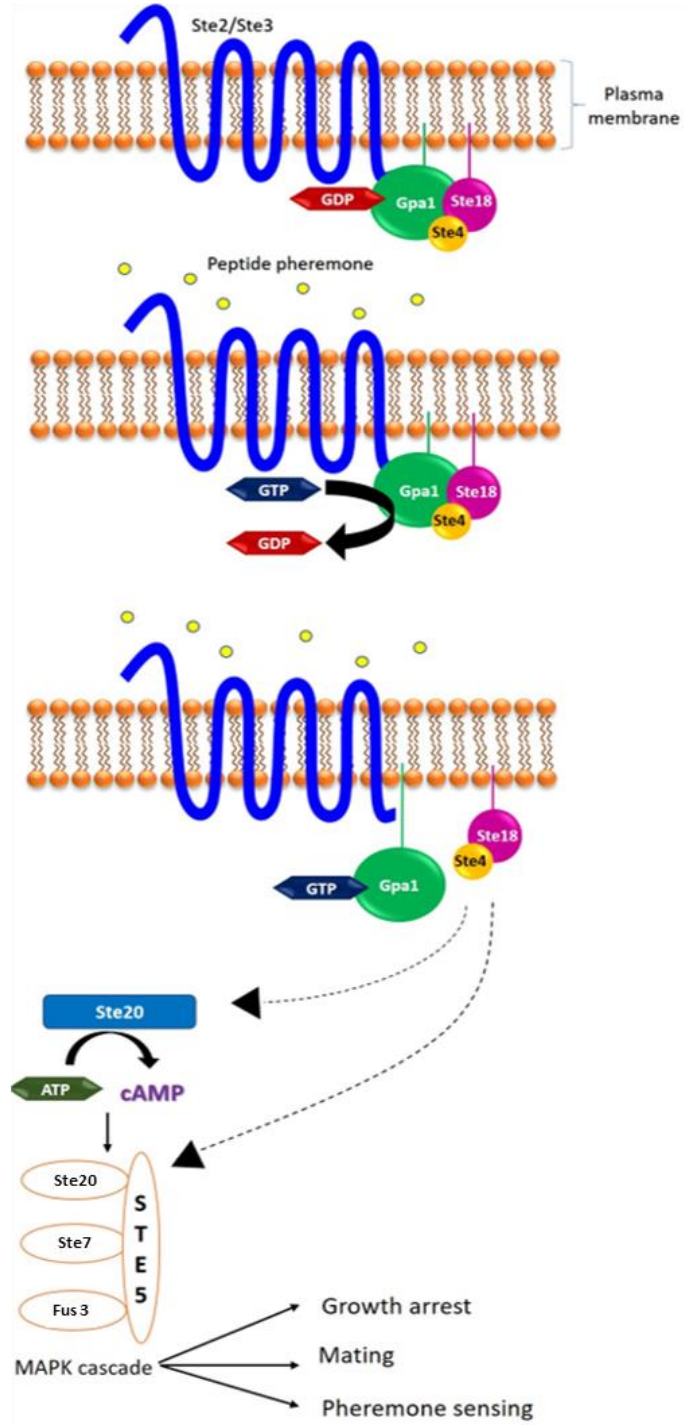

Figure 1: An overview of glucose and pheromone sensing GPCR signalling in yeast. a) Gpr1 activates Gpa2 upon ligand binding which subsequently activates Cde35/Cyr1. This leads to the downstream activation of cAMP-dependent protein kinase pathway which involves PKA-R and PKA-C that regulate responses for various developmental and mating processes. b) Ste2/Ste3 activates Gpa1 to dissociate from Ste18 and Ste4 dimer. Ste4 and Ste18 recruits Ste5 and Ste20 to the membrane and activate MAP kinase cascade (Ste11, Ste7 and Fus3) for mating process. 
Malays. J. Microbiol. Vol 14(6) Special Issue 2018, pp. 611-623 DOI: http://dx.doi.org/10.21161/mjm.1461823

\section{Other mechanisms}

\section{Host sensing}

Fungal pathogenesis is a biological process that involves the infection and development of disease in a host due to fungi. Diseases caused by fungal pathogens have resulted in devastating crop losses and has imposed alarming threat to human health. $M$. oryzae, a plant pathogenic fungus is believed to utilise GPCR mediated signalling to detect plant cell and start infection on them. The GPCR Pth11 in $M$. oryzae senses hydrophobic plant surfaces and promotes invasive growth. After the Pth11 receptor is activated through ligand binding, G-proteins will be dissociated and the $\beta y$ dimer activates adenylyl cyclase MAC I and MAPK pathway (Mst 11, Mst 7, Pmk 1) for appressorium formation and virulence (Kou et al., 2017). Another pathogenic fungus known as Fusarium oxysporum can sense host factors such as haemcontaining plant peroxidases in the roots before imposing attack on the plant host. The binding of the host factors to the sex pheromone receptor Ste2 activates the Mpk1 MAPK pathway for pathogenesis (Turrà et al., 2015). These well-characterised pathways may serve as a guide to study the host sensing pathways mediated by GPCR in other pathogenic fungi.

\section{Amino acid sensing}

Amino acids are building blocks of proteins which constitute most living tissue. It is also a source of nitrogen which is required for various developmental processes in fungi and to survive in nutrient-deficient condition. Gpr4 in $C$. neoformans, functions as amino acid sensor and senses methionine and stimulates capsule formation and mating filament through cAMP-PKA signalling. Gpr4 interacts with Gpa1 and the cyclase associated protein Aca1 to activate adenylyl cyclase (Cac1) to regulate downstream elements of the CAMP pathway which leads to the binding of CAMP to the regulatory subunit of PKA (Pkr1) and release of the PKA catalytic subunits (Pka1/2) (Xue et al., 2006). In other eukaryotes like animals, the amino acids are sensed by class C GPCR, for example, ligand binding of amino acids to GPRC6A receptor activates phospholipase C (PLC) effector and promotes secretion of GLP-1 in enterocyte L-cells to regulate insulin secretion (Rolland et al., 2001). The presence of pathways to sense amino acid via GPCR across the lower to higher eukaryotes shows that this mechanism is conserved to some level in all eukaryotes.

\section{Nutrient sensing}

Heterotrophic fungi depend on organic matter from other organisms for their nutrition, metabolism, and survival. To absorb these organic compounds from the environment, fungi must be able to sense them, and this is possible through GPCR signalling mechanism. In C. albicans, the Gpr1 receptor senses nutrients such as lactate, glucose and methionine and subsequently causes the dissociation of $\mathrm{G}$ protein. The dissociated Gpa2 activates the effector, Cyr1 which stimulates the cAMP-PKA pathway. The PKA activates Efg1 (transcription factor) to induce cell wall biosynthesis, filamentation, adhesion and biofilm formation (Dijck, 2009). Unlike this pathway, the Gpr1 involved in glucose sensing mechanism described earlier in $S$. cerevisiae is only activated by glucose and not amino acid or any other nutrient.

\section{Comparison of fungal GPCR with other eukaryotes}

In this portion we would like to elucidate if there are any differences in the role played or the mode of action amongst GPCRs in eukaryotic organisms. The following section will dissect the similarities and dissimilarities that have been observed for us to understand the diversity in the functionality of this group of proteins within the eukaryotes.

Fundamentally, all the GPCRs found in fungi and other eukaryotes such as plants and animals, share the same structure that is the portrayal of seven transmembrane (7TM) regions (Katritch et al., 2013). G proteins have three subunits: $G \alpha, G \beta$, and $G y$. The GPCRs undergo conformational change due to the binding of ligand which triggers the $G$ proteins to dissociate and in turn activates the effectors to initiate intracellular signalling responses. The wealth of evidence and knowledge accumulated in animal systems on GPCR has made them the ideal system to explain the GPCRs in other organisms. However, it's hard to ignore the fact that GPCRs in other systems are different in their own way and may conduct signalling in certain manner which can be slightly different in accordance to the physiological processes required for the survival of a particular group of organisms. The GPCRs in fungi are not well represented as compared to GPCRs from the other eukaryote system, but recent studies have provided the importance of GPCRs specifically to fungi. Through a series of inquiries below, we believe that the information derived will provide a better understanding of GPCRs and also serve to direct future research in this area.

\section{How do the G-proteins differ in function between the eukaryotes?}

In terms of the G-proteins, there are no reports hitherto stating that fungal GPCRs are structurally different from those in other eukaryotes. Nevertheless, the specific function of the G-protein differs across organisms. In fungi, $G$ proteins are required for growth, asexual and sexual development, and virulence ( $\mathrm{Li}$ et al., 2007). To conduct these functions, the GPCRs are specifically required to sense light, nutrient, carbon, nitrogen and pheromones (Xue et al., 2008). On the other hand, for other eukaryotes system, for example human and animals, the G-proteins are needed to regulate various cellular behaviours to the majority of neurotransmitters and hormones within the complex human and animal body (Heng et al., 2013). As for plants, G proteins are important in some physiological roles such as control of 
Malays. J. Microbiol. Vol 14(6) Special Issue 2018, pp. 611-623 DOI: http://dx.doi.org/10.21161/mjm.1461823

stomatal aperture, defence against biotic stress, oxidative stress, developmental process, sugar perception, and some phytochrome/cryptochrome-mediated responses (Urano and Jones, 2014). While there are clear differences, there are also some underlying similarities where plant, fungi and animals all require hormone/pheromone sensing and nutrient sensing for survival. Therefore, from these already understood models, a leaf may be taken from them to hypothesise, test and develop similar or alternative sensing pathways that may be present in fungi.

\section{Are all signalling mechanisms in eukaryotes GPCR dependent?}

The one striking similarity between animal and fungal signalling is that both organisms are GPCR-dependent. However, that is not the case for plants as they are GPCR independent. Hydrolysis of GTP and G-protein dissociation is caused by GPCR in animal and fungi where as in plants the G-proteins are activated by the regulator of G-protein signalling protein (RGS) (Urano et al., 2012). Despite this, both GPCR dependent and GPCR independent signalling promotes the activation of effector systems for downstream signalling cascades for various physiological processes. Our immediate question would be if fungi are solely dependent on GPCR for Gprotein signalling? Intriguingly, a study indicates that fungi may not fully depend on GPCR, as an intracellular guanine nucleotide exchange factors (GEF) found in $S$. cerevisiae is proposed to function in a same manner as the activator of $G$ protein-signalling (AGS) which are thought to compete with GPCR to activate the G-protein signalling (Lee and Dohlman, 2008). Hence, more studies involving gene knockout or gene silencing is required to verify if signalling via G-protein can be mediated without GPCR across all the representative member of each phylum in fungi. Table 2 shows brief differences of GPCR in fungi and other eukaryotes.

\section{How vast is the occurrence of the G-protein subunits in eukaryotes?}

As compared to animals, G-protein subunits characterised in plants and fungi are scarce. In fungi, specifically in yeast, two a subunits were characterised whereas the most characterised filamentous fungi possess three $\alpha$ subunits that are constituted of different classes (Bölker, 1998; Kays and Borkovich, 2004; Li et al., 2007). Nearly all the fungi possessed a single predicted $\beta$ subunit, but recently in $R$. oryzae, it was predicted to have four $\beta$ subunits (Li et al., 2007). Filamentous fungi have a single y subunit (Krystofova and Borkovich, 2005) but other fungal species like $C$. neoformans, $C$. cinereus, Podospora anserina, and $R$ oryzae possess more than one $\mathrm{y}$ subunit (Palmer et al., 2006). For each of the three subunits of the G-proteins, multiple family members existed (23 $\alpha, 5 \beta, 12 \gamma)$ in humans and animals and different combinations of these subunits allow specific G-protein based signalling pathways (Wettschureck and Offermanns, 2005; Trusov and Botella, 2016). Conversely, plants only have a single $\alpha$ (GPA1) and $\beta$ (AGB1) subunit and two $y$ subunits (AGG1 and AGG2) in Arabidopsis (Ma et al., 1990; Weiss et al., 1994; Mason and Botella, 2000; 2001). Through further genome studies and mining of information on fungal G-proteins and their subunits, more information may be obtained to help understand and delineate the signalling mechanism for different pathways.

\section{Do all the eukaryotes share the same effectors?}

The activation of G-protein leads to the consequent activation of effectors to generate intracellular signalling (Lodish et al., 2000). The main effectors of $G$ proteins acting downstream in filamentous fungi are the cAMPdependent and mitogen-activated protein kinase (MAPK) signalling cascades (Li et al., 2007; Gupta et al., 2014). Other specific effectors characterised in fungi includes adenylyl cyclases such as MAC 1 in $M$. grisea, Cac1 in $C$. neoformans, Cdc35 in $C$. albicans and Cyr1 in $S$. cerevisiae. As compared to fungi, the effector system in animal GPCR signalling are well defined and the signal is transmitted to various downstream effectors such as adenylyl cyclases, phospholipase C $\beta$, RGS-RhoGEFs (Wettschureck and Offermanns, 2005) and ion channels (Hille, 1992). The plant genome does not encode the effectors found in animal, however some protein interactors such as thylakoid formation 1 (THF1) and ACIreductone dioxygenase 1 may function in a similar manner to the canonical $G$ protein effectors (Jones and Assmann, 2004). Future research should emphasise on identifying other effectors besides adenylyl cyclase in fungi. Apart from that, analysis on sequence homology between the effectors found in fungi and effectors from other eukaryote system that are well characterised may provide a better understanding on how these effectors work similarly to conduct the downstream signalling.

Does ligand binding promote G-protein signalling differently across the eukaryotes?

Ligand binding induces the GPCR activation through the rearrangement of the transmembrane helices which triggers activation for downstream signalling with $G$ proteins and modulation of cellular physiology (Rosenbaum et al., 2009). In fungi, different ligands that bind to the GPCRs promote different intracellular signalling pathways. For example, mating-pheromone binds to Ste2/Ste3 for pheromone signalling whereas glucose binds to Gpr1 for glucose signalling. In animal systems, a chosen ligand can stimulate different intracellular signalling pathways independently through a single GPCR. In contrast, in plants, ligands appear to hamper the inhibitor in Arabidopsis whereby a ligand (Dglucose) impedes AtRGS1 GTPase-activating protein activity which allows AtGPA1 to be triggered without GPCR (Johnston et al., 2007). It would be interesting to investigate whether a single ligand can activate different signalling pathways in fungi like in the animal system. 
Malays. J. Microbiol. Vol 14(6) Special Issue 2018, pp. 611-623 DOI: http://dx.doi.org/10.21161/mjm.1461823

\section{CONCLUSION}

GPCRs are multi-functional receptors which are crucial in mediating responses and in regulating the receptor expression. Our review is centred on the studies conducted so far in fungal GPCRs including the classification, physiological roles, mechanism of action in GPCR signalling as well as the differences of GPCRs between fungi and other eukaryotes. Based on this review, we have identified a few gaps in knowledge of fungal GPCR research. We have listed below some questions that may be addressed through future research to enhance our knowledge in fungal GPCR.

i) Are fungi solely dependent on GPCR for G-protein signalling?

ii) What are the specific functions of the mammalian like GPCRs in fungi?

iii) How are the fungal specific and mammalian-like GPCRs involved in mechanism of action?

iv) How do the receptors like Stm 1 sense nitrogen starvation and how are microbial opsins involved in sensory function?

v) If GPCR can sense fatty acids, how does this mechanism work?

vi) Can a single ligand activate different signalling pathways in fungi like the animal system?

vii) Can there be any other effectors besides adenylyl cyclase in fungi?

viii) Is the GPCR diversity conserved in fungi?

ix) Are the fungal specific GPCRs domains well characterised?

$x)$ Is there any cross-talking in the GPCR signalling pathways of fungi?

Answering these questions may put together the missing pieces in the current knowledge about fungal GPCR. With further information and advancement in knowledge, we believe many key questions on how, where, and what of fungal GPCR may be determined to achieve the ultimate goal of having a well-defined and distinct characterisation of fungal GPCR system that stands out among other eukaryote systems. However, as we continue to answer these questions there will be others that crop up and thus ensue the continuous quest for more knowledge and information in this area.

\section{ACKNOWLEDGEMENTS}

This research was supported by funds from the Ministry of Science Technology and Innovation Malaysia (grant no: 02-01-02-SF0757 and 02-01-02SF1232) and DCP-2017$004 / 1$.

\section{REFERENCES}

Affeldt, K. J., Carrig, J., Amare, M. and Keller, N. P. (2014). Global survey of canonical Aspergillus flavus G protein-coupled receptors. mBio 5, e 01501-14.
Alspaugh, J. A., Perfect, J. R. and Heitman, J. (1997). Cryptococcus neoformans mating and virulence are regulated by the G-protein a subunit GPA1 and cAMP. Genes and Development 11, 3206-3217.

Bahn, Y. S., Xue, C., Idnurm, A., Rutherford, J. C., Heitman, J. and Cardenas, M. E. (2007). Sensing the environment: Lessons from fungi. Nature Reviews Microbiology 5, 57.

Beyer, M., Röding, S., Ludewig, A. and Verreet, J. A. (2004). Germination and survival of Fusarium graminearum macroconidia as affected by environmental factors. Journal of Phytopathology 152, 92-97.

Bieszke, J. A., Braun, E. L., Bean, L. E., Kang, S., Natvig, D. O. and Borkovich, K. A. (1999a). The nop-1 gene of Neurospora crassa encodes a seven transmembrane helix retinal-binding protein homologous to archaeal rhodopsins. Proceedings of the National Academy of Sciences 96, 8034-8039.

Bieszke, J. A., Spudich, E. N., Scott, K. L., Borkovich, K. A. and Spudich, J. L. (1999b). A eukaryotic protein, NOP-1, binds retinal to form an archaeal rhodopsin-like photochemically reactive pigment. Biochemistry 38, 14138-14145.

Bölker, M. (1998). Sex and crime: Heterotrimeric G proteins in fungal mating and pathogenesis. Fungal Genetics and Biology 25, 143-156.

Burkholder, A. C. and Hartwell, L. H. (1985). The yeast a-factor receptor: Structural properties deduced from the sequence of the STE2 gene. Nucleic Acids Research 13, 8463-8475.

Chaudhari, N., Landin, A. M. and Roper, S. D. (2000). A metabotropic glutamate receptor variant functions as a taste receptor. Nature Neuroscience 3, 113-119.

Chen, P., Sapperstein, S. K., Choi, J. D. and Michaelis, S. (1997). Biogenesis of the Saccharomyces cerevisiae mating pheromone a-factor. The Journal of Cell Biology 136, 251-269.

Choi, W. and Dean, R. A. (1997). The adenylate cyclase gene MAC1 of Magnaporthe grisea controls appressorium formation and other aspects of growth and development. The Plant Cell 9, 1973-1983.

Chung, K. S., Won, M., Lee, S. B., Jang, Y. J., Hoe, K. L., Kim, D. U., Lee, J. W., Kim, K. W. and Yoo, H. S. (2001). Isolation of a novel gene from Schizosaccharomyces pombe: Stm1+ encoding a seven-transmembrane loop protein that may couple with the heterotrimeric Ga2 protein, Gpa2. Journal of Biological Chemistry 276, 40190-40201.

Chung, K. S., Won, M., Lee, J. J., Ahn, J., Hoe, K. L., Kim, D. U., Song, K. B. and Yoo, H. S. (2007). Yeast-based screening to identify modulators of Gprotein signalling using uncontrolled cell division cycle by overexpression of Stm1. Journal of Biotechnology 129, 547-554.

Daynes, C. M., McGee, P. A. and Midgley, D. J. (2008). Utilisation of plant cell-wall polysaccharides and organic phosphorus substrates by isolates of Aspergillus and Penicillium isolated from soil. Fungal Ecology 1, 94-98. 
Malays. J. Microbiol. Vol 14(6) Special Issue 2018, pp. 611-623 DOI: http://dx.doi.org/10.21161/mjm.1461823

d'Enfert, C. (1997). Fungal spore germination: Insights from the molecular genetics of Aspergillus nidulans and Neurospora crassa. Fungal Genetics and Biology 21, 163-172.

Doehlemann, G., Berndt, P. and Hahn, M. (2006). Different signalling pathways involving a $\mathrm{Ga}$ protein, cAMP and a MAP kinase control germination of Botrytis cinerea conidia. Molecular Microbiology 59, 821-835.

de Mendoza, A., Sebé-Pedrós, A. and Ruiz-Trillo, I. (2014). The evolution of the GPCR signalling system in eukaryotes: Modularity, conservation, and the transition to metazoan multicellularity. Genome Biology and Evolution 6, 606-619.

Dijck, P. V. (2009). Nutrient sensing G protein-coupled receptors: Interesting targets for antifungals? Sabouraudia 47, 671-680.

Drake, M. T., Shenoy, S. K. and Lefkowitz, R. J. (2006). Trafficking of $\mathrm{G}$ protein-coupled receptors. Circulation Research 99, 570-582.

Fredriksson, R., Lagerström, M. C., Lundin, L. G. and Schiöth, H. B. (2003). The G protein-coupled receptors in the human genome form five main families. Phylogenetic analysis, paralogon groups, and fingerprints. Molecular Pharmacology 63, 1256-1272.

Fredriksson, R. and Schioth, H. B. (2005). The repertoire of $G$ protein-coupled receptors in fully sequenced genomes. Molecular Pharmacology 67(5), 1414-1425.

Galagan, J. E., Henn, M. R., Ma, L. J., Cuomo, C. A. and Birren, B. (2005). Genomics of the fungal kingdom: Insights into eukaryotic biology. Genome Research 15, 1620-1631.

Gomez-Diaz, C. and Benton, R. (2013). The joy of sex pheromones. EMBO Reports 14, 874-883.

Gupta, V. G., Schmoll, M., Herrera-Estrella, A., Upadhyay, R., Druzhinina, I. and Tuohy, M. (2014). Biotechnology and Biology of Trichoderma. Elsevier Science and Technology, Oxford, United Kingdom.

Hagen, D. C., McCaffrey, G. and Sprague, G. F. (1986). Evidence the yeast STE3 gene encodes a receptor for the peptide pheromone a factor: Gene sequence and implications for the structure of the presumed receptor. Proceedings of the National Academy of Sciences 83, 1418-1422.

Han, K. H., Seo, J. A. and Yu, J. H. (2004a). Regulators of $\mathrm{G}$ protein signalling in Aspergillus nidulans: RgsA downregulates stress response and stimulates asexual sporulation through attenuation of $\mathrm{GanB}(\mathrm{G \alpha})$ signalling. Molecular Microbiology 53, 529-540.

Han, K. H., Seo, J. A. and Yu, J. H. (2004b). A putative $\mathrm{G}$ protein-coupled receptor negatively controls sexual development in Aspergillus nidulans. Molecular Microbiology 51, 1333-1345.

Heinbockel, T., Laaris, N. and Ennis, M. (2007). Metabotropic glutamate receptors in the main olfactory bulb drive granule cell-mediated inhibition. Journal of Neurophysiology 97, 858-870.

Heng, B. C., Aubel, D. and Fussenegger, M. (2013). An overview of the diverse roles of $G$ protein-coupled receptors (GPCRs) in the pathophysiology of various human diseases. Biotechnology Advances 31, 16761694.

Hille, B. (1992). G protein-coupled mechanisms and nervous signalling. Neuron 9, 187-195.

Huang, H. C. and Klein, P. S. (2004). The frizzled family: Receptors for multiple signal transduction pathways. Genome Biology 5, 234.

Idnurm, A. and Howlett, B. J. (2001). Characterization of an opsin gene from the ascomycete Leptosphaeria maculans. Genome 44, 167-171.

Idnurm, A. and Heitman, J. (2005). Light controls growth and development via a conserved pathway in the fungal kingdom. PLoS Biology 3, e95.

Johnston, C. A., Taylor, J. P., Gao, Y., Kimple, A. J. Grigston, J. C., Chen, J. G., Siderovski, D. P., Jones, A. M. and Willard, F. S. (2007). GTPase acceleration as the rate-limiting step in Arabidopsis G protein-coupled sugar signalling. Proceedings of the National Academy of Sciences 104, 17317-17322.

Jones, A. M. and Assmann, S. M. (2004). Plants: The latest model system for $G$ protein research. EMBO Reports 5, 572-578.

Jones, S. K. and Bennett, R. J. (2011). Fungal mating pheromones: Choreographing the dating game. Fungal Genetics and Biology 48, 668-676.

Katritch, V., Cherezov, V. and Stevens, R. C. (2013). Structure-function of the $\mathrm{G}$ protein-coupled receptor superfamily. Annual Review of Pharmacology and Toxicology 53, 531-556.

Kays, A. and Borkovich, K. (2004). Signal transduction pathways mediated by heterotrimeric $\mathrm{G}$ proteins. In: The Mycota, Vol III: Biochemistry and Molecular Biology. Brambl, R. and Marzluf, G. A. (eds.). Springer, New York, USA. pp.175-207.

Klein, P. S., Sun, T. J., Saxe, C. L., Kimmel, A. R., Johnson, R. L. and Devreotes, P. N. (1988). A chemoattractant receptor controls development in Dictyostelium discoideum. Science 241, 1467-1472.

Koelle, M. R. (2006). Heterotrimeric G protein signalling: Getting inside the cell. Cell 126, 25-27.

Kou, Y., Tan, Y. H., Ramanujam, R. and Naqvi, N. I. (2017). Structure-function analyses of the Pth11 receptor reveal an important role for CFEM motif and redox regulation in rice blast. New Phytologist 214, 330-342.

Krishnan, A., Almén, M. S., Fredriksson, R. and Schiöth, H. B. (2012). The origin of GPCRs: Identification of mammalian like rhodopsin, adhesion, glutamate and frizzled GPCRs in fungi. PloS One 7, e29817.

Krystofova, S. and Borkovich, K. A. (2005). The heterotrimeric G protein subunits GNG-1 and GNB-1 form a $G \beta y$ dimer required for normal female fertility, asexual development, and $\mathrm{Ga}$ protein levels in Neurospora crassa. Eukaryotic Cell 4, 365-378.

Krystofova, S. and Borkovich, K. A. (2006). The predicted G protein-coupled receptor GPR-1 is required for female sexual development in the 
multicellular fungus Neurospora crassa. Eukaryotic Cell 5, 1503-1516.

Kulkarni, R. D., Thon, M. R., Pan, H. and Dean, R. A. (2005). Novel G protein-coupled receptor-like proteins in the plant pathogenic fungus Magnaporthe grisea. Genome Biology 6, R24.

Lagerström, M. C. and Schiöth, H. B. (2008). Structural diversity of $G$ protein-coupled receptors and significance for drug discovery. Nature Reviews Drug Discovery 7, 339.

Lee, M. J. and DohIman, H. G. (2008). Coactivation of G protein signaling by cell-surface receptors and an intracellular exchange factor. Current Biology 18, 211. 215.

Lengeler, K. B., Davidson, R. C., D'souza, C., Harashima, T., Shen, W. C., Wang, P., Pan, X., Waugh, M. and Heitman, J. (2000). Signal transduction cascades regulating fungal development and virulence. Microbiology and Molecular Biology Reviews 64, 746-785.

Li, L., Wright, S. J., Krystofova, S., Park, G. and Borkovich, K. A. (2007). Heterotrimeric G protein signalling in filamentous fungi. Annual Review of Microbiology 61, 423-452.

Liu, H., Suresh, A., Willard, F. S., Siderovski, D. P., Lu, S. and Naqvi, N. I. (2007). Rgs1 regulates multiple $\mathrm{Ga}$ subunits in Magnaporthe pathogenesis, asexual growth and thigmotropism. The EMBO Journal 26, 690-700.

Lodish, H., Berk, A., Zipursky, S. L., Matsudaira, P., Baltimore, D. and Darnell, J. (2000). Molecular Cell Biology 4th edition. W. H. Freeman and Company. New York, USA.

Lorenz, M. C., Pan, X., Harashima, T., Cardenas, M. E., Xue, Y., Hirsch, J. P. and Heitman, J. (2000). The G protein-coupled receptor Gpr1 is a nutrient sensor that regulates pseudohyphal differentiation in Saccharomyces cerevisiae. Genetics 154, 609-622.

Ma, H., Yanofsky, M. F. and Meyerowitz, E. M. (1990). Molecular cloning and characterisation of GPA1, a G protein alpha subunit gene from Arabidopsis thaliana. Proceedings of the National Academy of Sciences 87, 3821-3825.

Maidan, M. M., De Rop, L., Serneels, J., Exler, S., Rupp, S., Tournu, H., Thevelein, J. M. and Van Dijck, P. (2005). The G protein-coupled receptor Gpr1 and the $\mathrm{Ga}$ protein $\mathrm{Gpa} 2$ act through the cAMPprotein kinase A pathway to induce morphogenesis in Candida albicans. Molecular Biology of the Cell 16, 1971-1986.

Maller, J. L. (2003). Fishing at the cell surface. Science 300, 594-595.

Mason, M. G. and Botella, J. R. (2000). Completing the heterotrimer: Isolation and characterisation of an Arabidopsis thaliana $\mathrm{G}$ protein $\mathrm{Y}$-subunit cDNA. Proceedings of the National Academy of Sciences 97, 14784-14788.

Mason, M. G. and Botella, J. R. (2001). Isolation of a novel $G$ protein $\mathrm{y}$-subunit from Arabidopsis thaliana and its interaction with $\mathrm{G} \beta 1$. Biochimica et Biophysica
Acta (BBA)-Gene Structure and Expression 1520, 147-153.

Miller, L. J., Dong, M. and Harikumar, K. G. (2012). Ligand binding and activation of the secretin receptor, a prototypic family BG protein-coupled receptor. British Journal of Pharmacology 166, 18-26.

Miwa, T., Takagi, Y., Shinozaki, M., Yun, C. W., Schell, W. A., Perfect, J. R., Kumagai, H. and Tamaki, H. (2004). Gpr1, a putative G protein-coupled receptor, regulates morphogenesis and hypha formation in the pathogenic fungus Candida albicans. Eukaryotic Cell 3, 919-931.

Overington, J. P., Al-Lazikani, B. and Hopkins, A. L. (2006). How many drug targets are there? Nature Reviews Drug Discovery 5, 993.

Padgett, C. L. and Slesinger, P. A. (2010). GABAB receptor coupling to G-proteins and ion channels. In: Advances in Pharmacology. Academic Press, Cambridge, Massachussetts. pp.123-147.

Palmer, D. A., Thompson, J. K., Li, L., Prat, A. and Wang, P. (2006). Gib2, a novel G $\beta$-like/RACK1 homolog, functions as a G $\beta$ subunit in cAMP signalling and is essential in Cryptococcus neoformans. Journal of Biological Chemistry 281, 32596-32605.

Panagiotou, G., Pachidou, F., Petroutsos, D., Olsson, L. and Christakopoulos, P. (2008). Fermentation characteristics of Fusarium oxysporum grown on acetate. Bioresource Technology 99, 7397-7401.

Plakidou-Dymock, S., Dymock, D. and Hooley, R. (1998). A higher plant seven-transmembrane receptor that influences sensitivity to cytokinins. Current Biology 8, 315-324.

Premont, R. T. and Gainetdinov, R. R. (2007). Physiological roles of $\mathrm{G}$ protein-coupled receptor kinases and arrestins. Annual Review of Physiology 69, 511-534.

Raisley, B., Zhang, M., Hereld, D. and Hadwiger, J. A. (2004). A cAMP receptor-like G protein-coupled receptor with roles in growth regulation and development. Developmental Biology 265, 433-445.

Rao, T. P. and Kühl, M. (2010). An updated overview on Wnt signalling pathways: A prelude for more. Circulation Research 106, 1798-1806.

Rolland, F., Winderickx, J. and Thevelein, J. M. (2001). Glucose-sensing mechanisms in eukaryotic cells. Trends in Biochemical Sciences 26, 310-317.

Rosenbaum, D. M., Rasmussen, S. G. and Kobilka, B. K. (2009). The structure and function of $G$ proteincoupled receptors. Nature 459, 356.

Schandel, K. A. and Jenness, D. D. (1994). Direct evidence for ligand-induced internalization of the yeast alpha-factor pheromone receptor. Molecular and Cellular Biology 14, 7245-7255.

Sgourakis, N. G., Bagos, P. G., Papasaikas, P. K. and Hamodrakas, S. J. (2005). A method for the prediction of GPCRs coupling specificity to G proteins using refined profile Hidden Markov Models. BMC Bioinformatics 6, 104.

Spudich, J. L., Yang, C. S., Jung, K. H. and Spudich, E. N. (2000). Retinylidene proteins: Structures and 
Malays. J. Microbiol. Vol 14(6) Special Issue 2018, pp. 611-623 DOI: http://dx.doi.org/10.21161/mjm.1461823

functions from archaea to humans. Annual Review of Cell and Developmental Biology 16, 365-392.

Stefan, C. J. and Blumer, K. J. (1994). The third cytoplasmic loop of a yeast $G$ protein-coupled receptor controls pathway activation, ligand discrimination, and receptor internalization. Molecular and Cellular Biology 14, 3339-3349.

Tang, X. I., Wang, Y., Li, D. I., Luo, J. and Liu, M. Y. (2012). Orphan G protein-coupled receptors (GPCRs): Biological functions and potential drug targets. Acta Pharmacologica Sinica 33, 363.

Trusov, Y. and Botella, J. R. (2016). Plant G proteins come of age: Breaking the bond with animal models. Frontiers in Chemistry 4, 24.

Tsitsigiannis, D. I. and Keller, N. P. (2006). Oxylipins act as determinants of natural product biosynthesis and seed colonization in Aspergillus nidulans. Molecular Microbiology 59, 882-892.

Turrà, D., El Ghalid, M., Rossi, F. and Di Pietro, A. (2015). Fungal pathogen uses sex pheromone receptor for chemotropic sensing of host plant signals. Nature 527, 521.

Tuteja, N. (2009). Signalling through G protein-coupled receptors. Plant Signalling and Behavior 4, 942-947.

Uddin, M. S., Kim, H., Deyo, A., Naider, F. and Becker, J. M. (2012). Identification of residues involved in homodimer formation located within a $\beta$-strand region of the $\mathrm{N}$-terminus of a yeast $\mathrm{G}$ protein-coupled receptor. Journal of Receptors and Signal Transduction 32, 65-75.

Urano, D., Phan, N., Jones, J. C., Yang, J., Huang, J., Grigston, J., Taylor, J. P. and Jones, A. M. (2012). Endocytosis of the seven-transmembrane RGS1 protein activates $G$ protein-coupled signalling in Arabidopsis. Nature Cell Biology 14, 1079.

Urano, D. and Jones, A. M. (2014). Heterotrimeric G protein-coupled signalling in plants. Annual Review of Plant Biology 65, 365-384.

Versele, M., Lemaire, K. and Thevelein, J. M. (2001). Sex and sugar in yeast: Two distinct GPCR systems. EMBO Reports 2, 574-579.

Waschuk, S. A., Bezerra, A. G., Shi, L. and Brown, L. S. (2005). Leptosphaeria rhodopsin: Bacteriorhodopsin-like proton pump from a eukaryote. Proceedings of the National Academy of Sciences of the United States of America 102, 6879-6883.

Weiss, C. A., Garnaat, C. W., Mukai, K., Hu, Y. and Ma, H. (1994). Isolation of cDNAs encoding guanine nucleotide-binding protein beta-subunit homologues from maize (ZGB1) and Arabidopsis (AGB1). Proceedings of the National Academy of Sciences 91, 9554-9558.

Wettschureck, N. and Offermanns, S. (2005). Mammalian $G$ proteins and their cell type specific functions. Physiological Reviews 85, 1159-1204.

Whiteway, M., Hougan, L., Dignard, D., Thomas, D. Y., Bell, L., Saari, G. C., Grant, F. J., O'Hara, P. and MacKay, V. L. (1989). The STE4 and STE18 genes of yeast encode potential $\beta$ and $\gamma$ subunits of the mating factor receptor-coupled G protein. Cell 56, 467-477.
Xue, Y., Batlle, M. and Hirsch, J. P. (1998). GPR1 encodes a putative $G$ protein-coupled receptor that associates with the Gpa2p Ga subunit and functions in a Ras independent pathway. The EMBO Journal 17, 1996-2007.

Xue, C., Bahn, Y. S., Cox, G. M. and Heitman, J. (2006). G protein-coupled receptor Gpr4 senses amino acids and activates the cAMP-PKA pathway in Cryptococcus neoformans. Molecular Biology of the Cell 17, 667-679.

Xue, C., Hsueh, Y. P. and Heitman, J. (2008). Magnificent seven: Roles of G-protein coupled receptors in extracellular sensing in fungi. FEMS Microbiology Reviews 32, 1010-1032.

Ying, S. H., Feng, M. G. and Keyhani, N. O. (2013). A carbon responsive $\mathrm{G}$ protein-coupled receptor modulates broad developmental and genetic networks in the entomopathogenic fungus, Beauveria bassiana. Environmental Microbiology 15, 2902-2921.

$\mathrm{Yu}, \mathrm{J}$. (2006). Heterotrimeric $\mathrm{G}$ protein signalling and RGSs in Aspergillus nidulans. The Journal of Microbiology 44, 145.

Yun, C. W., Tamaki, H., Nakayama, R., Yamamoto, K. and Kumagai, H. (1997). G protein-coupled receptor from yeast Saccharomyces cerevisiae. Biochemical and Biophysical Research Communications 240, 287292.

Yun, C. W., Tamaki, H., Nakayama, R., Yamamoto, K. and Kumagai, H. (1998). Gpr1p, a putative G proteincoupled receptor, regulates glucose-dependent cellular cAMP level in yeast Saccharomyces cerevisiae. Biochemical and Biophysical Research Communications 252, 29-33. 